\title{
Informatics at Russian Secondary School
}

\author{
Vladimir M. KIRYUKHIN ${ }^{1}$, Marina S. TSVETKOVA ${ }^{2}$ \\ ${ }^{1}$ Dept. of Informatics and Control Processes, National Research Nuclear University "MEPhI" \\ 31 Kashirskoe Shosse, Moscow 115409, Russian Federation \\ ${ }^{2}$ Academy of Improvement of Professional Skill and Professional Retraining of Educators \\ 8 Golovinskoe Shosse, Moscow 125212, Russian Federation \\ e-mail:vkiryukh@gmail.com,vkiryukhin@mephi.ru,ms-tsv@mail.ru
}

\begin{abstract}
Informatics" as an academic subject imparts a culture of information science and algorithms on a student; the ability to format and structure information, knowledge and experience using a variety of methods of data representation in relation to a particular task (lists, graphs, arrays, tables, schemes, graphics, diagrams and hierarchical structures) and using relevant programs of data processing; the idea of a computer as a universal information-processing device; the idea of basic concepts which they study: information, algorithms, models, and their properties. It develops algorithmic thinking necessary for professional activity in modern society; explains how concepts and constructs of informatics are applied in the real world, about the role of information technology and automated devices in people's lives, as well as in industry and scientific research; skills and abilities for safe and appropriate use of computers and internet networks, and the ability to observe the norms of communication, and operate ethically and within the law.
\end{abstract}

Keywords: informatics, computer science, curriculum in informatics at secondary school.

\section{Introduction}

In Russian schools, informatics as a subject has been included in the secondary and high schools since 1985 (Ershov et al., 1985). The first version of the subject was developed at the initiative of Academy of Sciences of the country. Since 1998 the first standard on informatics according to which all pupils of the country studied informatics from 8 to 11 grades as a separate subject has been introduced.

In 2010-2012 the new school educational standard for secondary and high schools (Minobrnauki of Russia, 2010) was developed in Russia. In this standard there is not only studying of informatics from 5 to 9 grades at the secondary school and at 10-11 classes of high school, but also a special program of IT studying of children is provided (a task - formation of IT of literacy) and design activity by pupils in integration with other school subjects (metasubject results of training). 
The textbooks on informatics for the secondary school join the content of studying which carries out both tasks. These subjects are invariant for schools, but the school can develop them and supplement (further it is italicized). To each subject the quantity of lessons (not less than 70 for a course) and the approximate content of studying is specified. An important place is allocated to an algorithmic component of contents that is very useful for olympiad training of talented school students already from the 5 th grade.

\section{Secondary School Informatics Curriculum}

The text shows possible study topics for students in the $5^{\text {th }}-6^{\text {th }}$ grade ( 1 hour a week, totalling 70 hours) and in the $7^{\text {th }}-9^{\text {th }}$ grade ( 1 hour a week, totalling 105 hours in the course). If a student continues the subject from the $5^{\text {th }}$ to $9^{\text {th }}$ grade, they will study a total of 180 hours. Supplementary course material not subject to assessment is indicated by italics.

\section{Topic "Informatics and Information Processes"}

\section{Section "Introduction"}

- $5^{\text {th }}-6^{\text {th }}$ grade ( 2 hours)

Information is one of the main general concepts in modern science.

There are different elements to the word "information": information in the form of data, which can be processed by an automated system, and information in the form of knowledge intended for human interpretation.

Types of information and data: texts, numbers, graphics and sound.

- $7^{\text {th }}-9^{\text {th }}$ grade (4 hours)

The history of the development of informatics as a science, and the work of Russian scientists. The concept of cybernetics. The ability to describe continuous objects and processes using discrete mathematics.

Information processes - processes connected with storage, conversion and transfer of data. Examples of information processes in technical and social areas, and in nature.

\section{Section "The computer as a universal data-processing device"}

- $5^{\text {th }}-6^{\text {th }}$ grade ( 2 hours)

Variety of computers. Information carriers used in ICT. Historical and future developments.

Hygiene, ergonomics and technical conditions of using ICT equipment.

- $7^{\text {th }}-9^{\text {th }}$ grade (4 hours)

Computer architecture: processor, RAM, external NVRAM, input/output devices; their specifications. 
Computers embedded in technical devices and production complexes. The robotising of production, additive technologies (3D-printers).

Software and hardware in a computer's operation.

Data volumes and access speeds characteristic of different types of carriers. Information carriers in nature.

Safety techniques and rules of computer use.

Economic, legal and ethical aspects of ICT use.

History and trends in computer development, improving computer characteristics. Supercomputers.

Physical limitations on computer characteristics.

Parallel computing.

\section{Topic "The Mathematical Basis of Informatics"}

\section{Section "Coding” (2 hours)}

- $7^{\text {th }}-9^{\text {th }}$ grade

Symbols. The alphabet is a finite quantity of symbols. Text is a finite sequence of symbols in a given alphabet. The quantity of different texts of a certain length in a certain alphabet.

Variety of languages and alphabets. Natural and formal languages.

Coding symbols of one alphabet using code words in another alphabet; code tables, decoding.

\section{Section "Numeral systems"}

- $5^{\text {th }}-6^{\text {th }}$ grade ( 2 hours)

Positional and non-positional numeral systems. Examples of representing numbers in positional numeral systems. Octal and hexadecimal numeral systems. Converting natural numbers from the decimal system to octal and hexadecimal systems and back again.

- $7^{\text {th }}-9^{\text {th }}$ grade (4 hours)

The base of a numeral system. The alphabet (plurality of digits) of a numeral system. Quantity of digits used in a numeral system with a particular base. Short and long forms of written numbers in positional numeral systems.

The binary numeral system, writing whole numbers within the range of 0 to 1024. Converting natural numbers from the decimal system to binary and binary to decimal.

Converting natural numbers from the binary system to the octal and hexadecimal system and back again.

Arithmetic in numeral systems.

Section "The binary alphabet" (4 hours)

- $7^{\text {th }}-9^{\text {th }}$ grade 
The representation of data in a computer as texts in a binary alphabet.

Binary code with a fixed codeword length. Code width - length of codeword. Examples of binary code with widths of 8, 16, 32 .

Units of measurement for binary text length: bit, byte, kilobyte, etc. The quantity of information contained in a message.

Kolmogorov's approach to determining the quantity of information.

The dependence of the quantity of combinations of code on code width. ASCII code. Coding the Cyrillic alphabet. Examples of coding letters in national alphabets. Standard Unicode. Code tables with an alphabet other than binary.

Distortion of code during transfer. Error-correcting codes. The capability of unique decoding for codes of various codeword length.

Section "Discretising” (3 hours)

- $7^{\text {th }}-9^{\text {th }}$ grade

Measurement and discretising. General digital representations of audiovisual and other continuous data.

Coding colours. Colour models. RGB and CMYK models. HSB and CMY models. Depth of coding. Acquaintance with raster and vector graphics.

Coding sound. Width and frequency of a recording. Quantity of recording channels.

Evaluating quantitative parameters connected with representation and storage of image and sound files.

\section{Section "Elements of combinatorics, set theory and mathematical logic"}

- $5^{\text {th }}-6^{\text {th }}$ grade ( 6 hours)

Sets. Specific quantities of elements in sets obtained from two or three base sets using union, intersection and addition operations.

Expressions. Simple and complex expressions. Euler-Venn diagrams. Logical values of expressions. Logical expressions. Logical operations: "and" (conjunction, logical multiplication), "or" (disjunction, logical addition), "is not" (logical negation).

- $7^{\text {th }}-9^{\text {th }}$ grade (6 hours)

Calculating the quantity of variants: formulae for multiplication and addition of variant quantity. Quantity of texts of a given length in a given alphabet.

Logical expressions. Rules of recording logical expressions. Logical operation priorities.

Truth tables. Building truth tables for logical expressions.

Logical sequential operations (material conditional) and logical equality operations. Properties of logical operations. Laws of algebraic logic. Use of truth tables to prove laws of algebraic logic.

Acquaintance with the logic foundations of a computer. The cell base of a computer. The history of cell bases. Logic cell circuits and their physical (electronic) realisation. 
Section "Lists, graphs and trees" (6 hours)

- $7^{\text {th }}-9^{\text {th }}$ grade

Lists. First element, final element, previous element, next element. Insertion, deletion and substitution of elements.

Graphs. Vertices, edges and paths. Directed and undirected graphs. The initial vertex (source) and final vertex (terminal) in a directed graph. Length (weight) of an edge and path. The concept of shortest path. Adjacency matrices of a graph (with edge lengths).

Trees. Roots, leaves and vertices (nodes). Earlier vertex, later vertex. Subtrees. Peripherals. Binary trees. Genealogical trees.

\section{Section "Executors and algorithms. Executor control"}

- $5^{\text {th }}-6^{\text {th }}$ grade ( 7 hours)

States, possible circumstances and system of executive commands; order commands and enquiry commands; executor refusal. The need formally to describe an executor. Manual executor control.

Executor control algorithms. A computer is an automatic device able to control executors to carry out commands according to a previously-assembled program. Computer control of an executor.

Computer control of a self-propelled robot. Compiling algorithms and programs to control an executor on a computer (robot, turtle etc.).

- $7^{\text {th }}-9^{\text {th }}$ grade (3 hours)

Signals. Feedback. Examples: a computer and an executor under their control (including a robot); a computer receiving a signal from a digital sensor during observations and experiments and controlling actual (including moving) devices.

Examples of robotised systems (a system controlling movement in a transport system, a welding line in a car factory, an automated home heating system, an autonomous transport control system etc.).

\section{Section "Robotics"}

- $5^{\text {th }}-6^{\text {th }}$ grade (15 hours)

Robotics is the science of developing and using automated technical systems. Autonomous robots and automated complexes. Microcontrollers. Signals. Feedback: receiving signals from digital sensors (touch, range, light, noise etc.).

Autonomous moving robots. Executor devices and sensors. Robot command system. Robot design. Robot modelling: command executor and control device. Manual and computer control of robots.

Example of a learning environment for developing control programs for moving robots. Control algorithms for moving robots. Realisation of "obstacle- avoidance", "follow-the-line" and other algorithms.

Analysis of robot-activity algorithms. Robot mechanism testing, debugging of robot-control program. Impact of measurement and calculation mistakes on the fulfilment of robot-control algorithms. 


\section{Topic "Algorithms and Elements of Programming"}

\section{Section "Algorithms"}

- 5th-6th grade (12 hours)

Verbal description of algorithms. Describing algorithms using flowcharts. Distinguishing verbal descriptions of algorithms from descriptions in formal algorithmic language.

Algorithmic constructs.

Sequence constructs. Linear algorithms. Limitations of linear algorithms: the inability to foresee the dependence of a sequence of actions to be fulfilled on source data.

Selection constructs. Conditional operator: full and non-full forms.

Fulfilment and non-fulfilments of a condition (true and false expressions). Simple and compound conditions. Compound condition recording.

Repeat constructs: cycles with a set number of repetitions, with a condition for fulfilment, with a cycle variable. Checking of fulfilment conditions of a cycle before and after the cycle body: post-and preconditions of the cycle. Cycle invariants.

Recording algorithmic constructs in a selected programming language.

- $7^{\text {th }}-9^{\text {th }}$ grade (12 hours)

An algorithmic language (programming language) is a formal language for writing algorithms. A program is an algorithm written in a specific algorithmic language.

Examples of written commands of selection, repeat and other constructs in different algorithmic languages.

Programming systems. Means of creating and executing a program.

\section{Section "Development of algorithms and programs" (18 hours)}

- $7^{\text {th }}-9^{\text {th }}$ grade

Concepts of a program's development stages: compiling a program's requirements, selecting an algorithm and realising it in the form of a program in a chosen algorithmic language, debugging the program using a chosen programming system, and testing.

The simplest techniques of dialogue debugging programs (selecting a breakpoint, executing it step-by-step, revising the values of the variables, post-mortem debugging).

Acquaintance with program documentation. Compiling descriptions of programs by sample.

Examples of program development.

Operators.

Representations of data structures.

Constants and variables. Variable: name and value. Types of variables: integer, real, symbolic, string, and logic. Tabular values (arrays). Single-dimensional arrays. Two-dimensional arrays. 
Subprograms.

Examples of data processing problems:

o Identifying the smallest and largest number from two, three or four supplied numbers.

- Identifying all roots of a quadratic equation.

- Populating a number array in relation to a formula or numerical input path.

- Identifying the sum of elements in a given finite number sequence or array.

- Identifying the smallest (largest) element in an array.

Acquaintance with algorithms to solve these tasks. Realising these algorithms in a chosen programming environment.

Acquaintance with arrangements of more complex data-processing problems and the algorithms of their solutions: array sorting, element-wise operations with arrays; processing of integers represented in decimal and binary numeric systems, identifying the largest common denominator (Euclid's algorithm) etc.

\section{Section "Analysis of algorithms" (2 hours)}

- $7^{\text {th }}-9^{\text {th }}$ grade

Complexity of an algorithm: time to execute the program and number of operations performed, memory usage; reliance on the size of source data. Examples of short programs process a small volume of data over many steps; examples of short programs processing a large volume of data.

Determining possible algorithm results in a given set of input data; determining possible input data leading to this result. Examples of describing objects and processes using a selection of digital characteristics, as well as interdependencies of said characteristics expressed by means of a formula.

\section{Section "Mathematical modelling"}

- $5^{\text {th }}-6^{\text {th }} \operatorname{grade}(5$ hours $)$

Concept of mathematical models.

Problems solved using mathematical (computer) modelling. Distinguishing mathematical models from full-scale models and verbal (literal) descriptions of objects.

- $7^{\text {th }}-9^{\text {th }}$ grade $(5$ hours $)$

Using computers while working with mathematical models. Computer experiments.

Examples of mathematical (computer) model usage when solving technical problems. Representation of a modelling cycle: building a mathematical model, its realisation on a computer, simple example checking (testing), carrying out computer experiments, analysing the results, refining models. 


\section{Topic "Using Computer Programs and Services"}

\section{Section "File system"}

- $5^{\text {th }}-6^{\text {th }}$ grade ( 2 hours)

Principles of building file systems. Catalogues (directories). Fundamental operations for working with files: creating, editing, copying, moving, deleting. Types of files.

- $7^{\text {th }}-9^{\text {th }}$ grade ( 3 hours)

Typical sizes of different types of files: a page of text, a whole book, a minutelong video, a one-and-a-half-hour film, a file of astronomical observations data, a temporary information file when mathematically modelling complex physical processes, etc.

File manager.

File system search.

Archiving and dearchiving.

Overview of programs. Data compression concepts. Work with archivers.

\section{Section "Preparing texts"}

- $5^{\text {th }}-6^{\text {th }}$ grade (6 hours)

Text documents and their structural elements (page, paragraph, line, word, character).

Methods of textual input using a scanner, textual recognition software, speech recognition software. Machine translation.

Spellcheckers and dictionaries.

- $7^{\text {th }}-9^{\text {th }}$ grade (6 hours)

Word processor - an instrument for creating, editing and formatting texts. The properties of a page, paragraph, character. Style formatting.

Including lists, tables and graphics in a text document. Including diagrams, formulae, page numbers, headers and footers, links etc. in a text document. Track changes.

The concept of a system of information, library and publishing standards. Business correspondence, academic publications, collaborative work. Abstract and footnotes.

\section{Section "Computer presentation"}

- $5^{\text {th }}-6^{\text {th }}$ grade (4 hours)

Preparing computer presentations.

- $7^{\text {th }}-9^{\text {th }}$ grade (3 hours)

Including audiovisual objects in the presentation. Using templates. Display controls of a presentation.

\section{Section "Graphic editing"}

- $5^{\text {th }-6^{\text {th }}}$ grade ( 4 hours) 
Acquaintance with graphic editing.

Inserting images using different digital devices (digital cameras and microscopes, video-cameras, scanners etc.).

- $7^{\text {th }}-9^{\text {th }}$ grade (4 hours)

Raster and vector graphics.

Editing graphic objects: altering size, compressing an image, cropping, rotating, inverting, working with particular areas (highlight, copy and fill colour), altering colour, brightness and contrast. Acquaintance with photo processing. Geometrical and stylistic transformations.

Means of computer projecting. Blueprints and working to them. Base operations: highlighting, joining, geometrical transformation of fragments and components. Diagrams, plans and maps.

Section "Electronic (dynamic) tables" (4 hours)

- $7^{\text {th }}-9^{\text {th }}$ grade

Electronic (dynamic) tables. Formulae using absolute, relative and mixed addressing; transforming formulae when copying. Highlighting table range and ordering (sorting) its elements; building graphs and diagrams.

Section "Databases. Managing requests" (3 hours)

- $7^{\text {th }}-9^{\text {th }}$ grade

Databases. A table as a representation of relationships. Building a data search request in a ready-prepared database. Links between tables.

Section "Work in the information space. Information and communication technologies"

- $5^{\text {th }}-6^{\text {th }}$ grade ( 3 hours)

Computer networks. The internet. Internet addresses. Domain name system. Websites.

Types of internet network activity. Internet services: e-mail, reference services (maps, planners etc.), search engines, software update services etc.

- $7^{\text {th }}-9^{\text {th }}$ grade ( 6 hours)

Means and methods of information searches. Constructing enquiries; browsers. Computer encyclopaedias and dictionaries. Computer maps and other reference systems.

Network data storage. More data in nature and technology (genomic data, results of physical experiments, internet data, in particular data on social networking sites). Technologies of processing and storing it.

Techniques to improve internet safety. Computer viruses and other malware; defending against them.

Problems of authenticating obtained information. Electronic signatures, certified sites and documents. Methods of individual and collective packaging of information on the internet. Interaction on computer networks: e-mail, chat, forums, teleconferences etc. 
Social communication on the internet. Personal information, and means of protecting it. Organisation of personal information space on a network.

Basic trends in the future development of ICT.

Standards in the field of informatics and ICT. Standardisation and standards in the field of informatics and ICT before the computer age (numerals, alphabets of national languages etc.) and in the computer age (programming language, addressing, internet networks etc.)

\section{Conclusion}

Informatics as an academic subject imparts a culture of information science and algorithms on a student; the ability to format and structure information, knowledge and experience using a variety of methods of data representation in relation to a particular task (lists, graphs, arrays, tables, schemes, graphics, diagrams and hierarchical structures) and using relevant programs of data processing; the idea of a computer as a universal information-processing device; the idea of basic concepts which they study: information, algorithms, models, and their properties; it develops algorithmic thinking necessary for professional activity in modern society; explains how concepts and constructs of informatics are applied in the real world, about the role of information technology and automated devices in people's lives, as well as in industry and scientific research; skills and abilities for safe and appropriate use of computers and internet networks, and the ability to observe the norms of communication, and operate ethically and within the law.

The subject's place is determined by academic results and reflected in personal, interdisciplinary and subject-specific results.

Personal results of mastering the basic educational programme of secondary general education should reflect the ability to engage in communication in social and collaborative processes of educational, socially-beneficial, academic-research, creative and other activities.

Interdisciplinary results of mastering the basic educational programme of secondary general education should reflect the formation and development of competence when using information and communication technologies (hereon "ICT capabilities").

The twofold inclusion of informatics into the school curriculum is reflected in the two vectors of academic performance:

- The development of algorithmic and logical thought processes and of mathematical models, including:

- The creation of a culture of information science and algorithms.

- The basic concepts studied: information, algorithms, models and their properties.

- The development of algorithmic thinking seen as necessary for professional activity in modern society. 
- The development of the ability to compile and write an algorithm for a specific operator.

- An understanding of algorithmic constructs, logical values and operations.

○ A knowledge of one of the programming languages and fundamental algorithmic structures - linear, conditional and cyclic.

- The ability to format and structure information, select a method of data representation in relation to a particular task - tables, schemes, graphics or diagrams using relevant programs of data processing.

- The mastering of simple methods of presenting and analysing statistical data; the representation of statistical regularity in the real world and of various methods of their study, the depiction of the simplest probability models; the development of the ability to extract information represented in tables, diagrams and graphics, describing and analysing numerical data arrays using suitable statistical characteristics and using an understanding of the probability properties of environmental factors when making decisions.

- The representation of fundamental informational processes in real-life situations and on a computer:

- The representation of a computer as a universal information-processing device.

- The development of basic skills and abilities for using computer devices.

- The development of abilities to apply the studied concepts, results and methods for solving practical tasks and tasks in related disciplines by using a computer.

- The obtaining of skills and abilities for safe and appropriate use of computers and the internet, and the ability to observe the norms of communication, operate ethically and within the law.

The informatics course at secondary school is augmented with electronic training materials on the websites ${ }^{1},{ }^{2}$, and also electronic textbooks and manuals on course ${ }^{3},{ }^{4}$.

The course extends additional lessons according to the choice of school students, including on olympiad informatics (Kiryukhin and Tsvetkova, 2014). Content of preparation on olympiad informatics and the technique of such preparation has been presented earlier (Kiryukhin, 2007; Kiryukhin, 2010; Kiryukhin and Tsvetkova, 2011).

\footnotetext{
${ }^{1}$ http://www.sc-edu.ru

${ }^{2}$ http://www.fcior.edu.ru

${ }^{3}$ http://metodist.1bz.ru/authors/informatika/1

${ }^{4}$ http://metodist.lbz.ru/authors/informatika/2
} 


\section{References}

Ershov, A.P., Monahov, V.M., Beshenkov, S.A. (Eds.). (1985). Fundamentals of the Computer Science: Trial Textbook for the Secondary Schools (in two parts). Prosveschenie, Moscow. (In Russian). http://lib.mexmat.ru/books/65541

Kiryukhin, V. (2007). The modern contents of the Russian national Olympiads in informatics. Olympiads in Informatics, 1, 90-104.

Kiryukhin, V. (2010). Mutual influence of the National educational standard and Olympiad in Informatics contents. Olympiads in Informatics, 4, 15-29.

Kiryukhin, V., Tsvetkova, M. (2011). Preparing for the IOI trough developmental teaching. Olympiads in Informatics, 5, 44-57.

Kiryukhin. V., Tsvetkova, M. (2014). Informatics. Programs of Extracurricular Activities of the School Students Studying on Preparation for the All-Russian Olympiad in Informatics: Grades 5-11. Methodological Manual for a Teacher. BINOM, Laboratory of knowledge. (In Russian).

Minobrnauki of Russia (2010). Basic Educational Program Secondary School. The Ministry of Education and Science of Russia. (In Russian). Retrieved April 13, 2016, from

http: //xn--80abucjiibhv9a.xn--p1ai/\%D0\%BF\%D1\%80\%D0\%BE \%D0\%B5\%D0\%BA $\%$ D $1 \% 82 \%$ D $1 \% 8 B / \% D$ $1 \% 84 \%$ D0 $\%$ B3 $\%$ D0 $\%$ BE $\%$ D $1 \% 81-\%$ D0 $\%$ B8- $\%$ D0 $\%$ BF $\%$ D0 $\%$ BE $\%$ DO $\%$ BE $\%$ D0 $\%$ BF
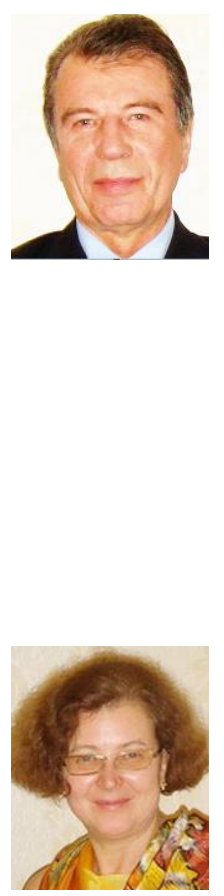

V.M. Kiryukhin is professor of the Russian Academy of Natural Sciences. He is the chairman of the federal methodical commission on informatics which is responsible in Russia for carrying out the national Olympiads in informatics. He is the author of many papers and books in Russia on development of Olympiad movements in informatics and preparations for the Olympiads in informatics. He is the exclusive representative who took part at all IOI from 1989 as a member of the IOI International Committee (1989-1992, 1999-2002, 2013-2016) and as the Russian team leader (1989, 1993-1998, 2003-2012). He received the IOI Distinguished Service Award at IOI 2003, the IOI Distinguished Service Award at IOI 2008 as one of the founders of the IOI making his long term distinguished service to the IOI from 1989 to 2008 and the medal "20 Years since the First International Olympiad in Informatics" at the IOI 2009.

M.S. Tsvetkova, professor of the Russian Academy of Natural Sciences, $\mathrm{PhD}$ in pedagogic science, prize-winner of competition "The Teacher of Year of Moscow" (1998), main expert of state projects of school education informatization in the Ministry of Education of the Russian Federation (2001-2005), the expert of the World bank project "Informatization of Education System". Since 2002 she is a member of the Central methodical commission of the Russian Olympiad in informatics, the pedagogic coach of the Russian team on the IOI. She is the author of many papers and books in Russia on the informatization of education and methods of development of talented students. Since 2013 she is the Russian team leader. 